\title{
Article \\ Towards Promising Platform by Using Annular Photonic Crystals to Simulate and Design Useful Mask
}

\author{
Ayman A Ameen ${ }^{1,2}$, Hussein A Elsayed ${ }^{1}$, Sagr Alamri ${ }^{3}$, Z.S. Matar ${ }^{4}$, M. Al-Dossari ${ }^{5}$ and Arafa H. Aly ${ }^{1, *}$ \\ 1 TH-PPM Group, Physics Department, Faculty of Science, Beni-Suef University, Beni Suef 62511, Egypt \\ 2 Physics Department, Faculty of Science, Sohag University, Sohag 82749, Egypt \\ 3 Department of Mechanical Engineering, College of Engineering, King Khalid University, \\ Abha 61421, Saudi Arabia \\ 4 Department of Physics, Faculty of Applied Science, Umm-Al-Qura University, Mecca 24381, Saudi Arabia \\ 5 Physics Department, Dhahran Aljanoub, King Khalid University, Abha 61421, Saudi Arabia \\ * Correspondence: arafa.hussien@science.bsu.edu.eg
}

Citation: Ameen, A.A.; Elsayed, H.A.; Alamri, S.; Matar, Z; Al-Dossari, M.; Aly, A.H. Towards Promising Platform by Using Annular Photonic Crystals to Simulate and Design Useful Mask. Photonics 2021, 8, 349. https:// doi.org/10.3390/photonics8090349

Received: 14 July 2021

Accepted: 19 August 2021

Published: 25 August 2021

Publisher's Note: MDPI stays neutral with regard to jurisdictional claims in published maps and institutional affiliations.

Copyright: () 2021 by the authors. Licensee MDPI, Basel, Switzerland. This article is an open access article distributed under the terms and conditions of the Creative Commons Attribution (CC BY) license (https:// creativecommons.org/licenses/by/ $4.0 /)$.

\begin{abstract}
Human masks are considered the mainstay in air filtration and purification technologies and against the spreading of bacterial and viral infections. This paper introduces a novel design of a human mask to increase the ultraviolet germicidal irradiation effect on pathogens. The proposed design consists of a tube with an annular photonic crystal (APC) attached to the mask's orifice, and a UV source is located in the tube's center. The main role of this study is the enhancement of UV doses based on the reflectivity of the proposed APC. Therefore, increasing pathogens' inactivation level in the incoming air to the mask's orifice could be investigated. The numerical investigations demonstrated that the proposed APC could provide a complete photonic bandgap with a high reflectivity in the wavelength regime from 207 to $230 \mathrm{~nm}$. In addition, we have considered the roles of the thickness of layers, inner core radius, and the azimuthal number. Meanwhile, the results showed the ability to use a wide range of core radius values without almost any variations in the optical properties of the proposed design. Such results could grant the advantage of using this design by the manufacturing of human masks with different sizes besides the inclusions in other ultraviolet germicidal irradiation applications.
\end{abstract}

Keywords: human masks; annular photonic crystals; photonic band gaps; transfer matrix method

\section{Introduction}

The spread of airborne-transmitted infections, such as the SARS-CoV-2 (COVID-19) outbreak, urgently highlights the need for personal protective equipment (PPE) to eliminate such biological hazards. One of the essential PPE is the human mask, as it can effectively prevent transferring aerosol pathogens via the respiratory route [1]. Masks contain spreading contaminated respiratory droplets generated through coughing and sneezing even through speaking [2]. In addition, they represent a physical barrier between aerosolized pathogens and the human respiratory system with various levels of protection [3]. Ultraviolet germicidal irradiation (UVGI) can be used to disinfect medical equipment and surgical masks. UVGI can effectively inactivate pathogens, as it destroys their genetic materials [4-7]. The level of inactivation depends on the type of the pathogen and ultraviolet (UV) doses as well. According to the FDA guidance, three log levels or more are required to accomplish an optimal inactivation dose [8]. The level of single-stranded RNA viruses reduces with the increase of UV dose [9]. UVGI devices, such as medical disinfectors, usually use $254 \mathrm{~nm}$ UV light, which has health hazards on the skin and eyes. An alternative approach to building UVGI could be obtained by using UV light in the range from 207 up to $222 \mathrm{~nm}$, which efficiently inactivates pathogens, without damaging human tissues or cells [10,11]. An optimal dose is required to achieve a sufficient inactivation level, which can be accomplished by a longer exposure time or a stronger UV source. 
Another way to increase UV dose with the same UV source and exposure time is to use an effective reflector. In this regard, photonic crystals (PCs) receive considerable attention due to its high reflectivity $[12,13]$. PCs are periodic arrangement structures with periodic refractive index functions that represent a building block in many optical, physical, and biomedical applications [14]. A range of electromagnetic frequencies is prohibited from propagating through PCs; this range is called the photonic bandgap (PBG) $[15,16]$. Bragg scattering at the interfaces of these periodic arrangements explains the physical interpretation of the PBG's appearance. PCs can be classified into one-dimensional, two-dimensional, and three-dimensional based on the structure periodicity, which leads to the formation of a PBG in a certain direction $[17,18]$. Although three-dimensional PCs express a complete PBG capable of reflecting electromagnetic waves in any direction, one-dimensional PCs are researched extensively with numerous designs due to the ease of fabrication $[19,20]$.

In this regard, researchers have used numerous methods to fabricate PCs, such as atomic layer deposition (ALD), chemical vapor deposition (CVD), and different lithographic techniques [12,21]. Recently, new methods for building PCs have been explored, for example, the self-assembly of nanoparticles to form colloidal PCs [22,23]. In particular, the ability of photon localization has been explored as a significant property of PCs. Such a property is promising in the design and experimental verifications of many technological applications in many different aspects. In this context, photon localization is formed by introducing a defect in this periodic arrangement, which causes a high transmittance peak inside the PBG $[24,25]$. Such a defect peak is sensitive to the change in the refractive index of the defect layer [26,27]. By using this feature, numerous sensors are designed to measure various conditions based on the changes in the refractive index under these conditions [28-33].

An alternative method to build two-dimensions PCs with a one-dimension periodicity is verified by using concentric cylindrical quarter-wavelength periodic structures. Such structures are known as annular photonic crystals (APCs), circular photonic crystals, or cylindrical Bragg reflectors $[34,35]$. APCs have attracted significant interest, since Kaliteevski et al., adopted the transfer matrix method (TMM) in cylindrical coordinates [36]. APCs offer low-loss (high Q-factor) resonators, making them suitable for chemical and biological sensing applications [37]. One of APCs' prime applications is the annular Bragg lasers, which can be integrated into optical fibers or other optical components for optoelectronics and optical communications [38]. APCs' desired properties, such as small modal volume, offer strong atom-photon interaction with applications including cavity quantum electrodynamics (QED) [39].

In this paper, we introduce a novel design based on APCs with high reflectivity or almost near-zero transmissivity in the wavelengths ranging from 207 to $222 \mathrm{~nm}$. The ability to investigate a PBG with high reflectivity in this range of wavelengths could efficiently inactivate pathogens without damaging human tissues or cells. Moreover, we choose polycarbonates as the material of the mask's tube, which is one of the most common materials for face mask production. Combining our structure with an active UV source for the disinfection of the proposed mask eliminates the need for disposable (single-use) face masks. Thus, the proposed design is eco-friendly, as it reduces plastic pollution coming from disposable masks. In this regard, such a design could be of interest to improve the performance of human masks against viral and bacterial infections. Our theoretical and numerical investigations are mainly based on the fundamentals of the TMM in cylindrical coordinates. Moreover, the effects of the periodicity number, the core radius, the thickness of constituent materials, and the azimuthal number are considered in detail.

\section{Theoretical Analysis}

In this section, we present an overview of the proposed mask and the basis of our theoretical modeling. The proposed mask consists of two parts: conventional parts like the mask's body, the rim that seals to the patient face, and the orifice. Non-conventional part: 
a tube attached to the mask's orifice coated by co-centric cylindrical multi-layers materials that form APC and UV source in its center as illustrated in Figure 1a. The incoming air enters the mask through the open end of a tube. As the air flows in the tube, it exposes to a suitable UV dose by the UV source and its reflection by the APC. The main purpose of APCs is to achieve a PBG in the wavelengths of interest with high reflectivity. Thus, an effective UV dose can be achieved without the need for a more powerful UV source that requires large-energy sources. Each unit cell in the proposed APCs consists of two cylindrical layers with refractive indices $n_{1}$ and $n_{2}$, respectively, and thicknesses $d_{1}$ and $d_{2}$, respectively, as shown in Figure $1 \mathrm{~b}$. The distance from the center of APC and $j$-th layer is defined as:

$$
\rho_{j}=\rho_{0}+\sum_{h=1}^{j} d_{h} \text { where, } j=1, \ldots, n,
$$

where $\rho_{0}$ is the radius of the air core layer with refractive index $n_{0}=1$. Here, each unit cell is repeated for a period number $N=\frac{n}{2}$, and the outer medium has a refractive index $n_{f}$. Our modeling is essentially based on the TMM in the cylindrical coordinates [36]. The two possible polarization modes for cylindrical Bragg waves are TE and TM polarizations. The non-zero fields at TE polarization modes are $E_{z}, H_{\phi}$, and $H_{\rho}$. The electric field $E_{z}$ and the magnetic field $H_{\phi}$ can be expressed as:

$$
\begin{gathered}
E_{z}(\rho, \phi)=V(\rho) \boldsymbol{\varphi}(\phi)=\left[A J_{m}(k \rho)+B Y_{m}(k \rho)\right] \exp (i m \phi), \\
H_{\phi}(\rho, \phi)=U(\rho) \boldsymbol{\varphi}(\phi)=-i p\left[A J_{m}^{\prime}(k \rho)+B Y_{m}^{\prime}(k \rho)\right] \exp (i m \phi),
\end{gathered}
$$

where $J_{m}$ and $Y_{m}$ are Bessel function and Numann function, respectively, and $J_{m}^{\prime}$, and $Y_{m}^{\prime}$ are their respective derivatives; the intrinsic admittance of the material is given as $p=\sqrt{\mu / \varepsilon}$, while the wave number inside the material is described as $k=\omega \sqrt{\mu \varepsilon} ; A$ and $B$ are constants, and $m$ is the azimuthal number. Then, the connection between $U(\rho)$ and $V(\rho)$ functions at the interface between two adjacent layers, such that $j$ th and $(j-1)$ th layers can be described by the following expression:

$$
\left[\begin{array}{l}
V\left(\rho_{j}\right) \\
U\left(\rho_{j}\right)
\end{array}\right]=M_{j}\left[\begin{array}{c}
V\left(\rho_{j-1}\right) \\
U\left(\rho_{j-1}\right)
\end{array}\right]
$$

where the matrix $M_{j}$ is given as:

$$
M_{j}=\frac{\pi}{2} k_{j} \rho_{j-1}\left[\begin{array}{ll}
m_{11} & m_{12} \\
m_{21} & m_{22}
\end{array}\right],
$$

where the elements of the matrix $M_{j}$ can be described as:

$$
\begin{gathered}
m_{11}=Y_{m}^{\prime}\left(q_{j-1}\right) J_{m}\left(q_{j}\right)-J_{m}^{\prime}\left(q_{j-1}\right) Y_{m}\left(q_{j}\right), \\
m_{12}=\frac{i}{p_{j}}\left[J_{m}\left(q_{j-1}\right) Y_{m}\left(q_{j}\right)-Y_{m}\left(q_{j-1}\right) J_{m}\left(q_{j}\right)\right], \\
m_{21}=-i p_{j}\left[Y_{m}^{\prime}\left(q_{j-1}\right) J_{m}^{\prime}\left(q_{j}\right)-J_{m}^{\prime}\left(q_{j-1}\right) Y_{m}^{\prime}\left(q_{j}\right)\right], \\
m_{22}=J_{m}\left(q_{j-1}\right) Y_{m}^{\prime}\left(q_{j}\right)-Y_{m}\left(q_{j-1}\right) J_{m}^{\prime}\left(q_{j}\right) .
\end{gathered}
$$

In the above expression, we have $q_{j-1}=k_{j} \rho_{j-1}$ and $q_{j}=k_{j} \rho_{j}$. The whole APC structure can be described by matrix $M$, which relates the fields at the inner core interface with radius $\rho_{0}$ to the outer medium $\rho_{f}$. Such matrix $M$ could be described as:

$$
\left[\begin{array}{c}
V\left(\rho_{f}\right) \\
U\left(\rho_{f}\right)
\end{array}\right]=M\left[\begin{array}{c}
V\left(\rho_{0}\right) \\
U\left(\rho_{0}\right)
\end{array}\right],
$$


where matrix $M$ is the product of the individual layers matrix, which can be written as:

$$
M=M_{2 N} M_{2 N-1} \ldots M_{1}=\left[\begin{array}{ll}
M_{11} & M_{12} \\
M_{21} & M_{22}
\end{array}\right] .
$$

Therefore, the reflectance of the proposed structure can be calculated in the vicinity of Equation (11) as following:

$$
R=\left|\frac{\left(M_{21}+i p_{0} C_{m 0}^{(2)} M_{11}\right)-i p_{f} C_{m f}^{(2)}\left(M_{22}+i p_{0} C_{m 0}^{(2)} M_{12}\right)}{\left(-i p_{0} C_{m 0}^{(1)} M_{11}-M_{21}\right)-i p_{f} C_{m f}^{(2)}\left(-j p_{0} C_{m 0}^{(1)} M_{12}-M_{22}\right)}\right|^{2}
$$

where $M_{11}, M_{11}, M_{12}, M_{21}$, and $M_{22}$ are the elements of the APC's matrix $M$, and $p=\sqrt{\varepsilon_{h} \mu_{h}}$, and $h=0$ and $f$ are for the inner core and the final media, respectively; $C_{m l}^{(1,2)}$ is obtained by the following equation:

$$
C_{m l}^{(1,2)}=\frac{H_{m}^{\prime(1,2)}\left(k_{l} \rho_{l}\right)}{H_{m}^{(1,2)}\left(k_{l} \rho_{l}\right)}, l=0, f
$$

where $H_{m}^{(1)}$ and $H_{m}^{(2)}$ are the first and the second kinds of Hankel function, respectively. The equations for $T M$ polarization mode can be acquired by replacing every $\varepsilon \leftrightarrow \mu$ and $i \leftrightarrow-i$ in all equations of $T E$ polarization mode.
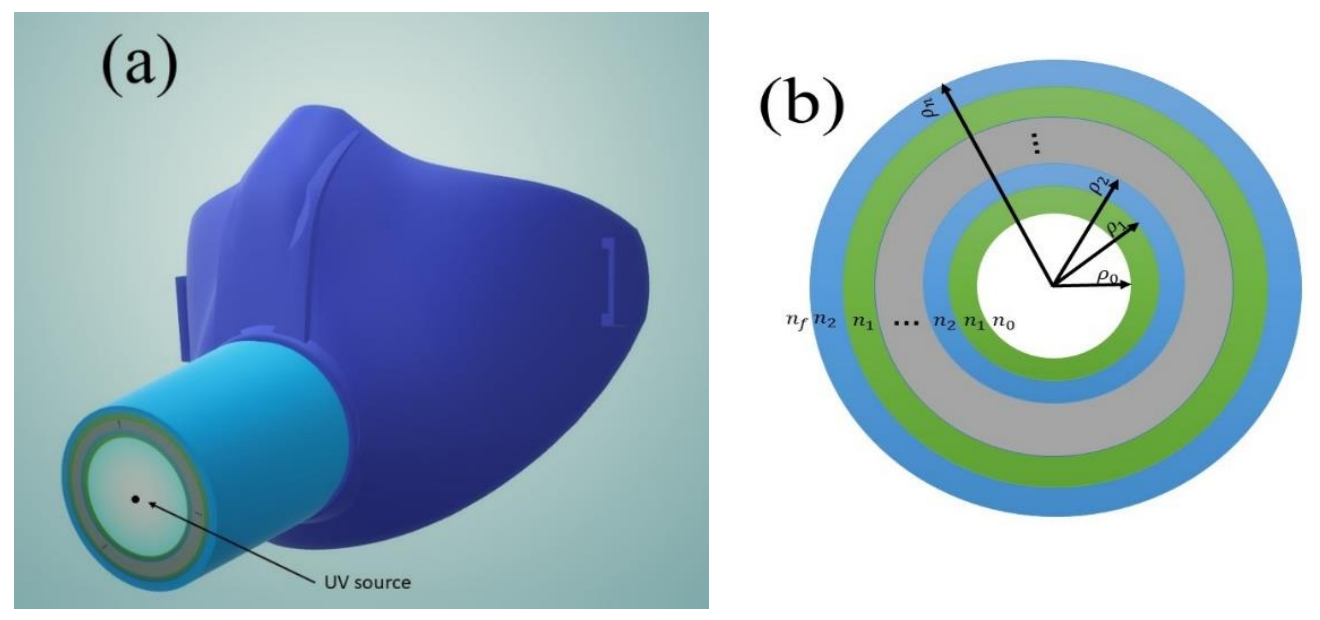

Figure 1. Schematic diagram of the proposed design including: (a) the mask design where the mask's body is sealed to the face; and (b) diagram of a planer view of the annular photonic crystal (APC) inside the mask with an internal radius $\rho_{0}$ where air enters the tube and concentric periodic cylinders with radii $\rho_{i}$.

\section{Results and Discussions}

In this section, we present the results of our modeling of the proposed APC with a UV source in the center of the mask. The proposed APC structure is embedded into a polycarbonates tube, with a real refractive index $n_{f}$ taken from reference [40] and an inner air core with refractive index $n_{0}=1$, as shown in Figure 1a. Polycarbonate is selected, due to its wide use in face masks, light weight, and reasonable cost [41]. The APC consists of a bi-layer unit cell repeated for periodicity number $N$, as illustrated in Figure $1 \mathrm{~b}$. The first layer in each unit cell is an $A l_{2} \mathrm{O}_{3}$ layer with thickness $d_{1}$ and refractive index $n_{1}$ which is taken form reference [42]. The second layer is composed of a $\mathrm{SiO}_{2}$ layer with thickness $d_{2}$ and refractive index $n_{2}$, taken from reference [43]. The initial parameters for the proposed APC are $d_{1}=29 \mathrm{~nm}, d_{2}=33.5 \mathrm{~nm}, N=20, \mathrm{~m}=0$, and $\rho_{0}=5 \mathrm{~cm}$. Frist, we studied the reflectance of our structure for TE polarization, as illustrated in Figure 2. 
The reflectance spectrum shows the appearance of the PBG between 207.7 and $229.6 \mathrm{~nm}$ with an average reflectance intensity around $94 \%$ and a central wavelength of $218 \mathrm{~nm}$. Moreover, the reflectivity at the center of the PBG reaches $99 \%$. The high reflectance promises a significant increase in UV dose without the need of more powerful UV sources or longer exposure time. Here, the location of the PBG covers the entire far-UVC light range of 207-222 nm, which efficiently inactivates pathogens without harmful effects on human tissues or cells [44]. Moreover, the harmful ozone production by UV light is deficient, as the absorption of UV light by oxygen in the chosen UV range is very low [45]. In this regard, the incident UV radiation could interact with the proposed design with its full intensity and almost no dissipation due to the production of the harmful Ozone. In particular, the mainstay of our study depends on the presence of an optimal dose to achieve a sufficient inactivation level.

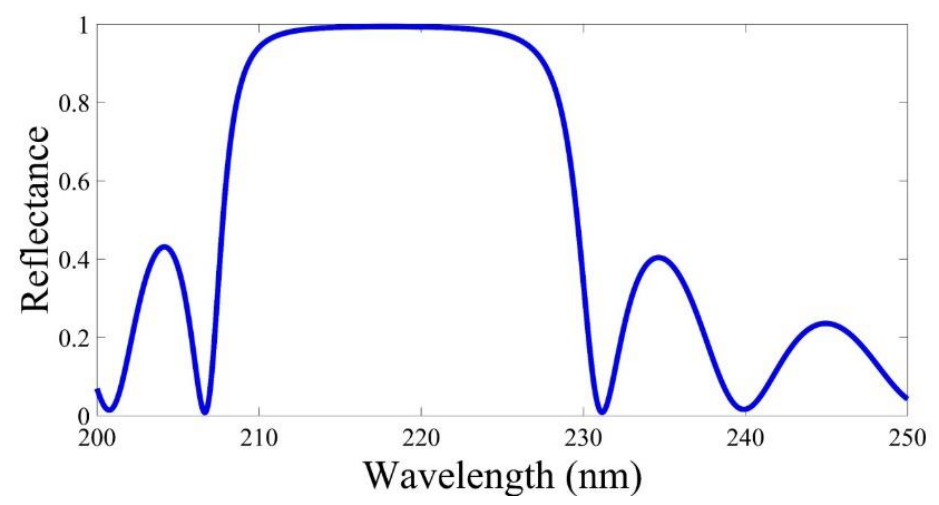

Figure 2. The reflectance of the proposed APC with air core radius $\rho_{0}=5 \mathrm{~cm}$ and unit cell layers' thicknesses $d_{1}=29 \mathrm{~nm}, d_{2}=33.5 \mathrm{~nm}$, and periodicity number $N=20$.

We considered the effects of altering the azimuthal number on the reflectance properties and the PBG of the proposed structure for both TE and TM polarizations. Figure 3 shows the reflectance of the APC structure, as the azimuthal number changes from 1 up to 20. The reflectance was calculated at an inner core radius $\rho_{0}=5 \mathrm{~cm}$ for both TE polarization (left) and TM polarization (right) with unit cell layer thicknesses $d_{1}=29 \mathrm{~nm}$ and $d_{2}=33.5 \mathrm{~nm}$ and periodicity number $N=20$. The reflectivity of our structure and the properties of its PBG are almost unaffected by the changes in the values of the azimuthal number for both TE and TM polarizations, as shown in Figure 3a-c. Therefore, the investigated result could grant our design another advantage over traditional multilayer structures. In particular, the optical properties of the traditional one-dimensional PCs are significantly affected with the changes in the values the angle of incidence, especially in reducing the PBG width for TM polarization. Here, we could avoid such a problem. In particular, the changes in the values of the azimuthal number have no effects on the optical properties of our design, especially at large values of the core inner radius. Therefore, the choice of APCs in the designs and fabrications of the human masks could be of increased concern.

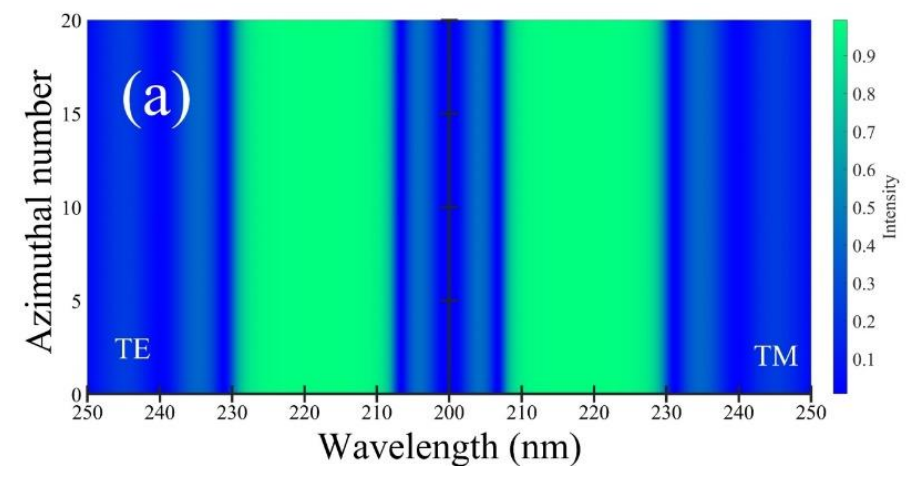

Figure 3. Cont. 

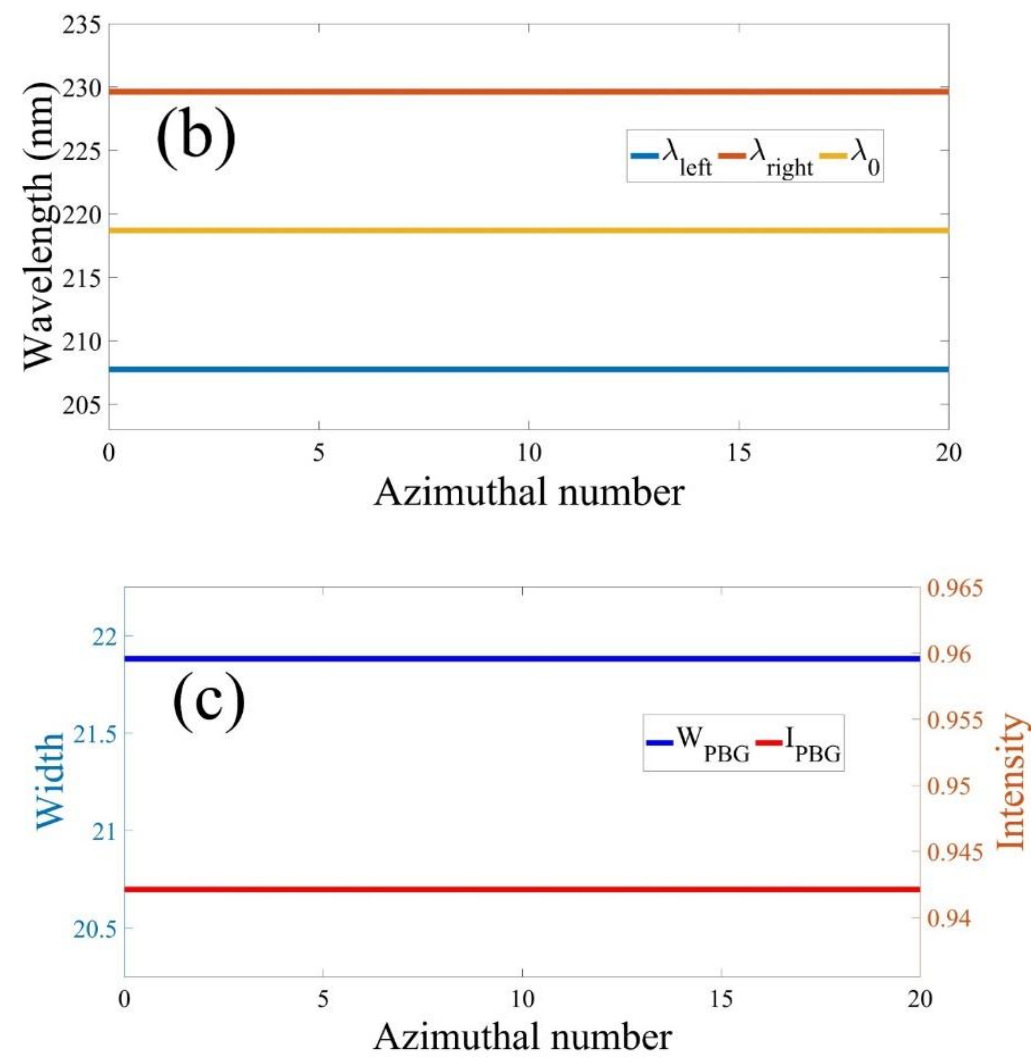

Figure 3. The effects of the azimuthal number for TE and TM polarizations on the reflectance of the proposed design (a), the edges and the central wavelength of the photonic bandgap (PBG) (b), and the average intensity $I_{P E G}$ and the width $W_{P E G}$ of the PBG (c).

Next, we discussed the effect of changing the thicknesses of the constituent materials on the reflectivity of our APCs, as shown in Figure 4. Here, the values of other related parameters are as following: the inner core radius $\rho_{0}=5 \mathrm{~cm}$, azimuthal number $m=0$, and periodicity number $N=20$. Figure 4 a illustrates the reflectance of the suggested APC structure as the thickness of $\mathrm{Al}_{2} \mathrm{O}_{3}$ layer $d_{1}$ changes from 29 up to $35 \mathrm{~nm}$ and the thickness of the $\mathrm{SiO}_{2}$ layer is fixed at $33.5 \mathrm{~nm}$. The increase of the $\mathrm{Al}_{2} \mathrm{O}_{3}$ layer thickness leads to a significant shift in the position of the PBG towards the longer wavelengths with a little increase in its width. However, the average intensity declines slightly with increasing the value of $d_{1}$. The left and the right edges of the PBG also shift towards longer wavelengths as well as the PBG's central wavelength. Then, we considered the effect of changing the thickness of the $\mathrm{SiO}_{2}$ layer $d_{2}$ on the reflectance of the proposed structure, as illustrated in Figure $4 \mathrm{~b}$. Thickness $d_{2}$ varies from 31 to $37 \mathrm{~nm}$, while the thickness of $A l_{2} \mathrm{O}_{3}$ layer $d_{1}$ is fixed at $29 \mathrm{~nm}$. Similar to the effect of $d_{1}$, the PBG is shifted towards the longer wavelengths with the increase in the value of $d_{2}$. Thus, the effect of thickness could be of interest in providing the different regions of UV sources with high reflectivity. 

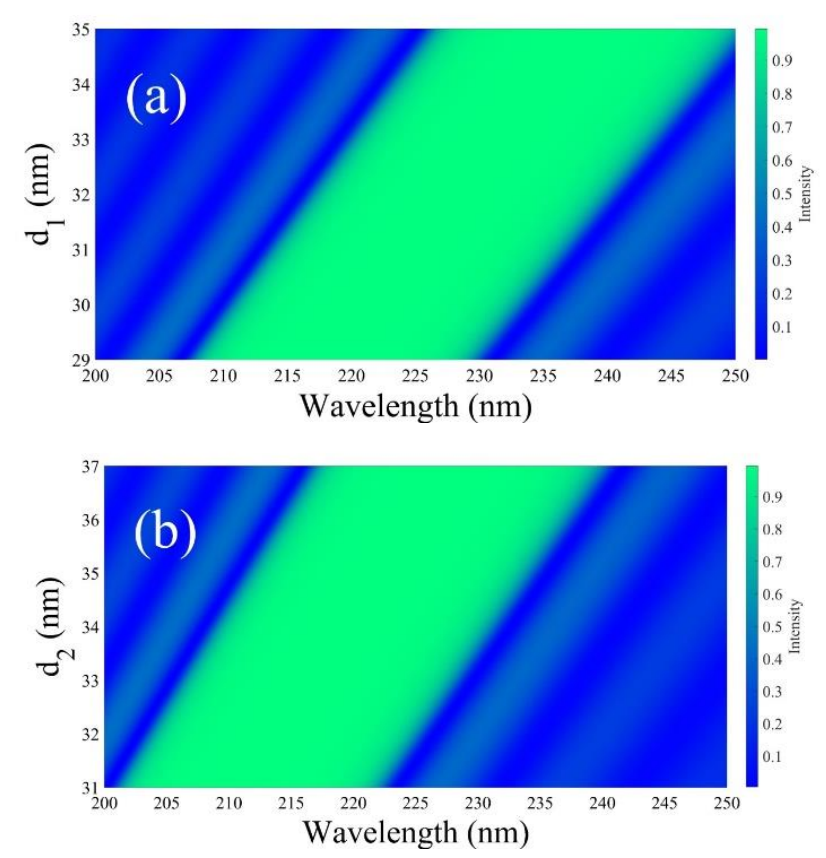

Figure 4. The influence of altering the thicknesses of the unit cell's layers on the structure reflectivity; (a) the thickness of $\mathrm{Al}_{2} \mathrm{O}_{3} ;\left(\right.$ b) the thickness of $\mathrm{S}_{\mathrm{i}} \mathrm{O}_{2}$.

Then, we analyzed the effects of varying $d_{1}$ and $d_{2}$ on the PBG's width. The simulation data, which describe the relationship between the width and thicknesses $d_{1}$ and $d_{2}$, can be fitted as following:

$$
W_{P B G}=0.41 d_{1}+0.35 d_{2}-2.03,
$$

where $W_{P B G}$ is the width of the PBG in nanometers at $A l_{2} \mathrm{O}_{3}$ layer thickness $d_{1}$ in nanometers and $\mathrm{SiO}_{2}$ layer thickness $d_{2}$ in nanometers. Equation (14) shows that the PBG's width grows with increasing either the values of $d_{1}$ or $d_{2}$ and the increase in the PBG's width becomes more prominent with increasing $d_{1}$ and $d_{2}$ simultaneously. Additionally, the PBG's central wavelength versus the thicknesses of the unit cell are fitted to the following equation:

$$
\lambda_{0}=3.2 d_{1}+2.8 d_{2}+27.9,
$$

where $\lambda_{0}$ is the PBG's central wavelength, and $d_{1}$ and $d_{2}$ are the unit cell layer's thicknesses. Equation (15) shows that the central wavelength of the PBG moves towards longer wavelengths with increasing both $d_{1}$ and $d_{2}$ or either of them; however, the shift in the PBG becomes larger with rising both $d_{1}$ and $d_{2}$ concurrently. Such effects, including the width of the PBG and its central wavelength, are as a result of the change in the optical path length of the incident radiation. In other words, the changes in the thicknesses of the constituent materials require a similar change in the position, width, and central wavelength of the PBG to verify the condition of the constant phase [45].

In addition, we investigated the effects of the number of periods on the reflectivity of our design for both TE and TM polarizations, as demonstrated in Figure 5. At low values of the number of periods especially smaller than 5, the PBG is not formed due to the limited chances of Bragg's scattering at the interfaces, as shown in Figure 5a. As the number of periods increases to 7, the PBG with little sharp edges begins to appear between 205 and $234 \mathrm{~nm}$, as shown in Figure 5a,b. For further increase in the number of periods, the edges of the PBG become sharper, and its width slightly decreases. At periodicity number $N=8$ for TE polarization, the PBG appears between 204.5 and $233.8 \mathrm{~nm}$ with a central wavelength of $219.17 \mathrm{~nm}$. Thus, the PBG's width equals $29.3 \mathrm{~nm}$, and its average intensity is $71.1 \%$. The PBG's width shrinks to $26 \mathrm{~nm}$ with an average intensity of $82 \%$, as the periodicity number reaches $N=11$. The PBG's intensity is approximately $98 \%$ with a width of $19 \mathrm{~nm}$, as the periodicity number increases to 40; meanwhile, the PBG central wavelength is almost 
stable at $218 \mathrm{~nm}$. Besides, the PBG parameters for TM polarization are almost identical to their values TE polarization, as shown in Figure 5b,c. Moreover, the peaks around the PBG become more intense and less wide, with a shift in their position towards the PBG as the periodicity number increases. Additionally, there is no significant difference between the reflectance in TE polarization and that in TM polarization with increasing the periodicity number. Then, the PBG's parameters were evaluated as written in Table 1. The PBG's left edge $\lambda_{\text {left }}$ is shifted towards the longer wavelengths while the right edge $\lambda_{\text {right }}$ moves to shorter wavelengths, as the periodicity number increases. The PBG's right and left edges were calculated when the reflectance's intensity reaches $50 \%$ of the maximum intensity in the PBG, while the PBG average intensity $I_{P E G}$ was calculated by the normalized sum of all simulation points between the right and left edges of the PBG. Therefore, the periodicity number can be adjusted to optimize the intensity of reflectance and the ease of fabrication.
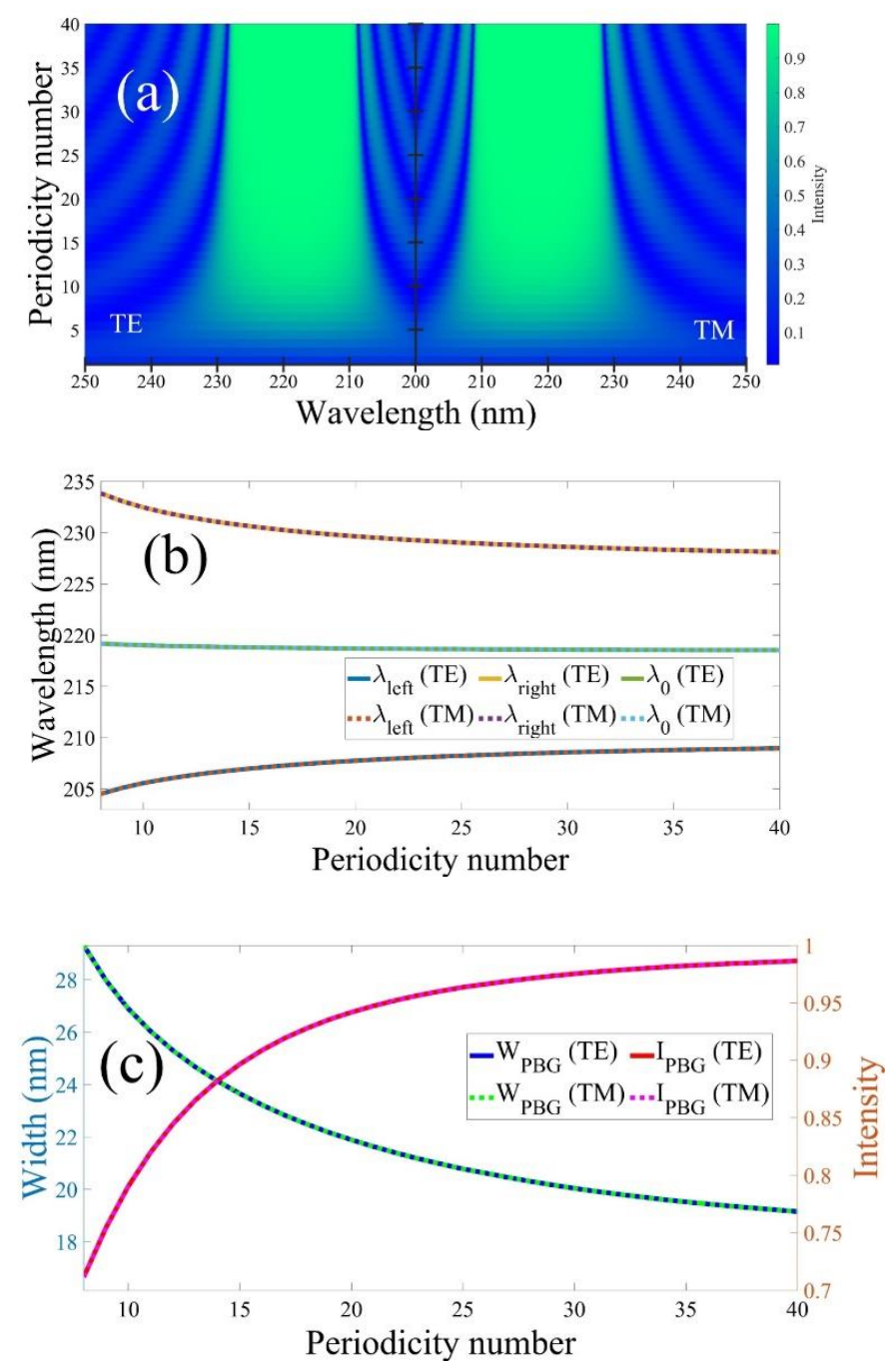

Figure 5. The effect of periodicity number for TE and TM polarizations on the reflectivity of the proposed APC (a), the left edge $\lambda_{\text {left }}$, right edge $\lambda_{\text {right }}$, and central wavelength $\lambda_{0}$ of the PBG (b), and the PBG's width $W_{P E G}$ and average intensity $I_{P B G}(\mathbf{c})$.

Table 1. The impact of the periodicity number on the PBG parameters.

\begin{tabular}{cccccc}
\hline $\boldsymbol{N}$ & $\lambda_{\text {left }}(\mathbf{n m})$ & $\lambda_{\text {right }}(\mathbf{n m})$ & $\lambda_{0}(\mathbf{n m})$ & $\boldsymbol{I}_{\text {PBG }}$ & $\boldsymbol{W}_{\text {PEG }}(\mathbf{n m})$ \\
\hline 15 & 206.9 & 230.6 & 218.8 & 0.89 & 23.6 \\
\hline 20 & 207.7 & 229.6 & 218.69 & 0.94 & 21.8 \\
\hline 25 & 208.2 & 229.0 & 218.63 & 0.96 & 20.7 \\
\hline
\end{tabular}


Finally, we have investigated the effect of inner core radius on the characteristics of the PBG and the reflectivity of our design for both TE and TM polarizations. Here, we have considered that, $N=20, d_{1}=29 \mathrm{~nm} d_{2}=33.5 \mathrm{~nm}$ and azimuthal number $m=0$. Figure 6 describes the effect of the inner core radius on the properties of the PBG and its reflectivity. Figure 6a illustrates the reflectance of the proposed APC at TE and TM polarizations with varying the inner core radius from $1 \mathrm{~cm}$ up to $30 \mathrm{~cm}$. The reflectance has no apparent changes, which adds more flexibility and simplicity from the fabrication point of view of human faces with different sizes. As the inner core radius changes from $1 \mathrm{~cm}$ up to $30 \mathrm{~cm}$, the structure reflectivity, width, and position of the PBG are almost unaffected, as shown in Figure 6b,c. Meanwhile, the PBG's central wavelength is located at $218.6 \mathrm{~nm}$ for both TE and TM polarizations and is almost unaffected by the increase in the values of the core radius. In particular, the increase in the core radius over a few micrometers is not almost effecting on the reflectivity of APC because the structure behaves like planer PCs and the intensity no longer depends on radius [35,46-48]. Thus, the reflectivity and the characteristic of the PBG of our structure provide a significant stability with the variations in the values of the inner core radius. Such result could be of potential interest from the experimental point of view in the manufacturing of human masks with different sizes to be compatible with the different faces. In addition, this result could open the way towards the inclusions of such design in many different applications that concern ultraviolet germicidal irradiation applications.
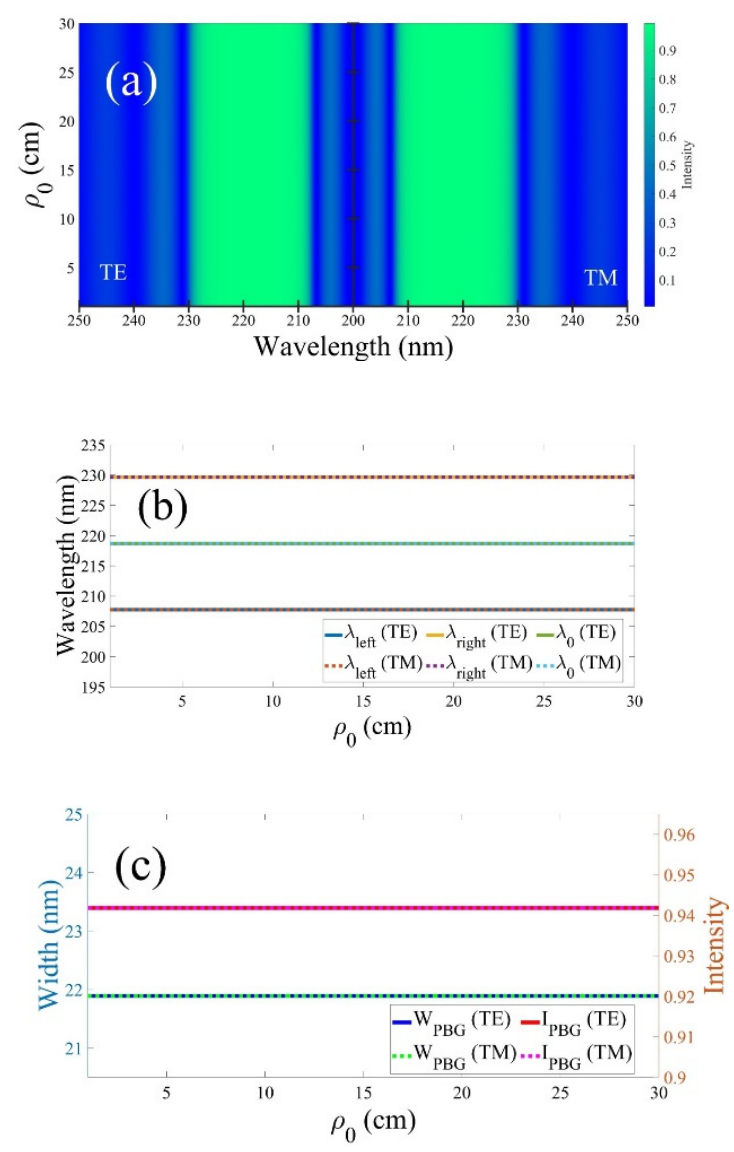

Figure 6. The influence of varying inner core radius for both $T E$ and $T M$ polarizations on the reflectance of the proposed APC (a), the PBG position (b), and the PBG's width $W_{P B G}$ and average intensity $I_{P E G}(\mathbf{c})$.

\section{Conclusions}

To sum up, we have introduced, in this research paper, a novel design of APCs with a high reflectivity in the wavelengths ranging from 207 to $230 \mathrm{~nm}$ and an average reflectance intensity of around $94 \%$. The proposed structure could be useful in producing 
an effective UV dose without the need for a more powerful UV source that requires highenergy sources. Therefore, the inclusion of such a design with human masks could leads to a significant improvement against viral and bacterial infections. In this context, the numerical results showed the appearance of a complete PBG with reflectivity is more than $99 \%$ at the center of the PBG. Moreover, the reflectivity and the PBG of the proposed design are almost unaffected by the azimuthal number change from 1 up to 20. Additionally, the effects of the core radius on the reflectivity and the PBG are negligible, as the inner core radius changes from 1 to $30 \mathrm{~cm}$. Therefore, the flexibility of using this structure through the different sizes of human masks is applicable. Moreover, the PBG could be easily shifted based on the variations of the thicknesses of the constituent materials, which allows the production of the different types of UV sources.

Author Contributions: Conceptualization, A.H.A. and A.A.A.; methodology, A.A.A.; software, A.A.A.; validation, A.A.A., H.A.E. and Z.S.M.; formal analysis, A.A.A.; investigation, H.A.E.; resources, S.A.; data curation, A.A.A.; writing-original draft preparation, A.A.A. and M.A.-D.; writing-review and editing, H.A.E., A.H.A. and M.A.-D.; visualization, A.H.A., A.A.A. and M.A.-D.; supervision, A.H.A.; project administration, A.H.A.; funding acquisition, S.A. and Z.S.M. All authors have read and agreed to the published version of the manuscript.

Funding: This research received no external funding.

Acknowledgments: The authors extend their appreciation to the Deanship of Scientific Research at King Khalid University, Saudi Arabia for funding this work through the Research Group Program under grant code number. R.G.P 2/127/42.

Conflicts of Interest: The authors declare that there are no conflict of interest.

\section{References}

1. Chua, M.H.; Cheng, W.; Goh, S.S.; Kong, J.; Li, B.; Lim, J.Y.; Mao, L.; Wang, S.; Xue, K.; Yang, L.; et al. Face Masks in the New COVID-19 Normal: Materials, Testing, and Perspectives. Research 2020, 2020, 1-40. [CrossRef]

2. Bahl, P.; Bhattacharjee, S.; de Silva, C.; Chughtai, A.A.; Doolan, C.; MacIntyre, C.R. Face coverings and mask to minimise droplet dispersion and aerosolisation: A video case study. Thorax 2020, 75, 1024-1025. [CrossRef]

3. Tang, J.W.; Liebner, T.J.; Craven, B.A.; Settles, G.S. A schlieren optical study of the human cough with and without wearing masks for aerosol infection control. J. R. Soc. Interface 2009, 6 (Suppl. 6), S727-S736. [CrossRef] [PubMed]

4. Weiss, M.M.; Weiss, P.D.; Weiss, D.E.; Weiss, J.B. Disrupting the Transmission of Influenza A: Face Masks and Ultraviolet Light as Control Measures. Am. J. Public Health 2007, 97 (Suppl. 1), S32-S37. [CrossRef]

5. Wilde, J.P.; Baer, T.M.; Hesselink, L. Modeling UV-C irradiation chambers for mask decontamination using Zemax OpticStudio. Appl. Opt. 2020, 59, 7596-7605. [CrossRef] [PubMed]

6. Shirbandi, K.; Barghandan, S.; Mobinfar, O.; Rahim, F. Inactivation of Coronavirus with Ultraviolet Irradiation: What? How? Why? SSRN 2020. [CrossRef]

7. Pecho, P.; Škvareková, I.; Ažaltovič, V.; Hrúz, M. Design of air circuit disinfection against COVID-19 in the conditions of airliners. Transp. Res. Procedia 2020, 51, 313-322. [CrossRef]

8. Center for Devices and Radiological Health. Enforcement Policy for Face Masks and Respirators During the Coronavirus Disease (COVID-19) Public Health Emergency (Revised). U.S. Food and Drug Administration. 10 July 2020. Available online: https: / / www.fda.gov/regulatory-information/search-fda-guidance-documents/enforcement-policy-face-masks-andrespirators-during-coronavirus-disease-covid-19-public-health (accessed on 22 February 2021).

9. Schnell, E.; Harriff, M.J.; Yates, J.E.; Karamooz, E.; Pfeiffer, C.D.; McCarthy, J.F.; Trapp, C.L.; Frazier, S.K.; Dodier, J.E.; Smith, S.M. Homegrown Ultraviolet Germicidal Irradiation for Hospital-Based N95 Decontamination during the COVID-19 Pandemic. medRxiv 2020. [CrossRef]

10. Aly, H.A.; Barakat, S.; Amin, A.F. Tunable filter based on the 1D photonic crystal within ultraviolet radiations. IOP Conf. Ser. Mater. Sci. Eng. 2020, 956, 012010. [CrossRef]

11. Welch, D.; Buonanno, M.; Grilj, V.; Shuryak, I.; Crickmore, C.; Bigelow, A.W.; Randers-Pehrson, G.; Johnson, G.W.; Brenner, D.J. Far-UVC light: A new tool to control the spread of airborne-mediated microbial diseases. Sci. Rep. 2018, 8, 1-7. [CrossRef]

12. Detchprohm, T.; Liu, Y.S.; Mehta, K.; Wang, S.; Xie, H.; Kao, T.T.; Shen, S.C.; Yoder, P.D.; Ponce, F.A.; Dupuis, R.D. Sub 250 nm deep-UV AlGaN/AlN distributed Bragg reflectors. Appl. Phys. Lett. 2017, 110, 011105. [CrossRef]

13. Ameen, H.; Elsayed, A.; Aly, H. Towards a highly efficient air purifier using annular photonic crystals in UV regimes. RSC Adv. 2021, 11, 14915-14921. [CrossRef]

14. Joannopoulos, J.D. (Ed.) Photonic Crystals: Molding the Flow of Light, 2nd ed.; Princeton University Press: Princeton, NJ, USA, 2008. 
15. Aly, H.; Elsayed, H.A. Defect mode properties in a one-dimensional photonic crystal. Phys. B Condens. Matter 2012, 407, 120-125. [CrossRef]

16. Aly, H.; Ameen, A.A.; Elsayed, H.A.; Mohamed, S.H.; Singh, M.R. One-Dimensional Metallo-Superconductor Photonic Crystals as a Smart Window. J. Supercond. Nov. Magn. 2019, 32, 2313-2318. [CrossRef]

17. Yue, Y.; Gong, J.P. Tunable one-dimensional photonic crystals from soft materials. J. Photochem. Photobiol. C Photochem. Rev. 2015, 23, 45-67. [CrossRef]

18. Dai, X.; Xiang, Y.; Wen, S.; He, H. Thermally tunable and omnidirectional terahertz photonic bandgap in the one-dimensional photonic crystals containing semiconductor InSb. J. Appl. Phys. 2011, 109, 053104. [CrossRef]

19. Moroz, A. Three-Dimensional Complete Photonic-Band-gap Structures in the Visible. Phys. Rev. Lett. 1999, 83, 5274-5277. [CrossRef]

20. Kazmierczak, T.; Song, H.; Hiltner, A.; Baer, E. Polymeric One-Dimensional Photonic Crystals by Continuous Coextrusion. Macromol. Rapid Commun. 2007, 28, 2210-2216. [CrossRef]

21. Graugnard, E.; Gaillot, D.P.; Dunham, S.N.; Neff, C.W.; Yamashita, T.; Summers, C.J. Photonic band tuning in two-dimensional photonic crystal slab waveguides by atomic layer deposition. Appl. Phys. Lett. 2006, 89, 181108. [CrossRef]

22. Guo, R.; Wang, D.-N.; Wei, Y.-Y.; Zhang, Y.-Z.; Yang, C.-G.; Xu, Z.-R. Colloidal photonic crystal array chip based on nanoparticle self-assembly on patterned hydrophobic surface for signal-enhanced fluorescent assay of adenosine. Microchim. Acta 2020, 187, 194. [CrossRef]

23. Qi, F.; Meng, Z.; Xue, M.; Qiu, L. Recent advances in self-assemblies and sensing applications of colloidal photonic crystals. Anal. Chim. Acta 2020, 1123, 91-112. [CrossRef]

24. Aly, H.; Elsayed, H.A.; Ameen, A.A.; Mohamed, S.H. Tunable properties of one-dimensional photonic crystals that incorporate a defect layer of a magnetized plasma. Int. J. Mod. Phys. B 2017, 31, 1750239. [CrossRef]

25. Aly, H.; Ameen, A.A.; Elsayed, H.A.; Mohamed, S.H. Photonic crystal defective superconductor and black body radiations. Opt. Quant. Electron. 2018, 50, 361. [CrossRef]

26. Aly, H.; Elsayed, H.A. Tunability of defective one-dimensional photonic crystals based on Faraday effect. J. Mod. Opt. 2017, 64, 871-877. [CrossRef]

27. King, T.-C.; Yang, Y.-P.; Liou, Y.-S.; Wu, C.-J. Tunable defect mode in a semiconductor-dielectric photonic crystal containing extrinsic semiconductor defect. Solid State Commun. 2012, 152, 2189-2192. [CrossRef]

28. Fenzl, C.; Hirsch, T.; Wolfbeis, O.S. Photonic Crystals for Chemical Sensing and Biosensing. Angew. Chem. Int. Ed. 2014, 53, 3318-3335. [CrossRef]

29. Mohamed, A.G.; Elsayed, H.A.; Mehaney, A.; Aly, A.H.; Sabra, W. The transmissivity of one-dimensional photonic crystals comprising three phases nanocomposite layer for optical switching purposes. Phys. Scr. 2021, 96, 115504. [CrossRef]

30. Seifouri, M.; Rouini, M.A.; Olyaee, S. Design of a surface plasmon resonance biosensor based on photonic crystal fiber with elliptical holes. Opt. Rev. 2018, 25, 555-562. [CrossRef]

31. Kehl, F.; Bischof, D.; Michler, M.; Keka, M.; Stanley, R. Design of a Label-Free, Distributed Bragg Grating Resonator Based Dielectric Waveguide Biosensor. Photonics 2015, 2, 124-138. [CrossRef]

32. Inan, H.; Poyraz, M.; Inci, F.; Lifson, M.A.; Baday, M.; Cunningham, B.T.; Demirci, U. Photonic crystals: Emerging biosensors and their promise for point-of-care applications. Chem. Soc. Rev. 2017, 46, 366-388. [CrossRef]

33. Seifouri, M.; Rouini, M.A.; Olyaee, S. A Photonic Crystal Fiber Based Surface Plasmon Resonance Biosensor with Elliptical and Circular Holes. J. Nanoelectron. Optoelectron. 2019, 14, 335-341. [CrossRef]

34. Chang, T.-W.; Hsu, H.-T.; Wu, C.-J. Investigation of Photonic Band Gap in a Circular Photonic Crystal. J. Electromagn. Waves Appl. 2011, 25, 2222-2235. [CrossRef]

35. Amiri, S.; Paul, B.K.; Ahmed, K.; Aly, A.H.; Zakaria, R.; Yupapin, P.; Vigneswaran, D. Tri-core photonic crystal fiber based refractive index dual sensor for salinity and temperature detection. Microw. Opt. Technol. Lett. 2019, 61, 847-852. [CrossRef]

36. Kaliteevski, M.A.; Abram, R.A.; Nikolaev, V.V.; Sokolovski, G.S. Bragg reflectors for cylindrical waves. J. Mod. Opt. 1999, 46, 875-890. [CrossRef]

37. Scheuer, J.; Yariv, A. Annular Bragg defect mode resonators. J. Opt. Soc. Am. B JOSAB 2003, 20, 2285-2291. [CrossRef]

38. Gu, J.; Xi, X.; Ma, J.; Yu, Z.; Sun, X. Parity-time-symmetric circular Bragg lasers: A proposal and analysis. Sci. Rep. 2016, 6, 1-7. [CrossRef] [PubMed]

39. Scheuer, J.; Green, W.M.J.; DeRose, G.A.; Yariv, A. InGaAsP annular Bragg lasers: Theory, applications, and modal properties. IEEE J. Sel. Top. Quantum Electron. 2005, 11, 476-484. [CrossRef]

40. Brissinger, D. Complex refractive index of polycarbonate over the UV-Vis-IR region from 0.2 to $3 \mu \mathrm{m}$. Appl. Opt. 2019, 58, 1341-1350. [CrossRef]

41. Fadare, E.D.; Okoffo, E.D. Covid-19 face masks: A potential source of microplastic fibers in the environment. Sci. Total Environ. 2020, 737, 140279. [CrossRef]

42. Weber, M.J. (Ed.) CRC Handbook of Laser Science \& Technology, 4: Optical Materials; Part 2; CRC Press: Boca Raton, FL, USA, 1986.

43. Weber, M.J. Handbook of Optical Materials; CRC Press: Boca Raton, FL, USA, 2003.

44. Buonanno, M.; Welch, D.; Shuryak, I.; Brenner, D.J. Far-UVC light (222 nm) efficiently and safely inactivates airborne human coronaviruses. Sci. Rep. 2020, 10, 1-8. [CrossRef]

45. Claus, H. Ozone Generation by Ultraviolet Lamps. Photochem. Photobiol. 2021, 97, 471-476. [CrossRef] [PubMed] 
46. Singh, S.; Singh, K. Design of an integrated multi-arm power splitter using photonic crystal waveguide. Optik 2017, 145, 495-502. [CrossRef]

47. Chen, M.-S.; Wu, C.-J.; Yang, T.-J. Optical properties of a superconducting annular periodic multilayer structure. Solid State Commun. 2009, 149, 1888-1893. [CrossRef]

48. Abadla, M.M.; Elsayed, H.A.; Mehaney, A. Thermo-Optical Properties of Binary One Dimensional Annular Photonic Crystal Including Temperature Dependent Constituents. Phys. E Low-Dimens. Syst. Nanostructures 2020, 119, 114020. [CrossRef] 